\title{
Pros and cons of polypharmacy in elderly patients with diabetes
}

\section{ABSTRACT}

According to the World Health Organization (WHO), polypharmacy is a safe and effective treatment with at least five drugs that is consistent with evidencebased medicine. Unfortunately, too often combination therapies are used without scientific justification. Contemporary available spectrum of hypoglycaemic drugs enables the use of a variety of combinations. Two or, less often, three drugs with different mechanisms of action are used simultaneously. Taking into account the fact that patients with T2DM are likely to have other diseases that require multiple medications, the potential risk of clinically relevant drug interactions is high. This may, inter alia, undesirably affect the daily fluctuations of glycaemia with the serious consequences of this phenomenon. The risk of severe hypoglycaemia or hyperglycaemia is especially related to elderly patients. In this group polypragmasy is much more common than in the same age group with normal carbohydrate metabolism. The aim of this paper is to discuss the phenomenon of polypragmasy with particular emphasis on its occurrence in elderly patients with T2DM. (Clin Diabetol 2017; 6, 1: 34-38)

Key words: polifarmacotheraphy, polypragmasia, diabetes mellitus, polifarmacotheraphy in diabetes

Address for correspondence:

lek. Dominik Wojtczak

e-mail: domixon1@gmail.com

Translation: lek. Małgorzata Kamińska

Clinical Diabetology 2017, 6, 1, 34-38

DOI: $10.5603 /$ DK.2017.0006

Received: 08.05.2017

Accepted: 30.05.2017

\section{Introduction}

Patients with type 2 diabetes mellitus (T2DM) are very often treated with two or three hypoglycaemic agents. This therapeutic strategy is based on the assumption that a combination of drugs with different mechanisms of action improves glycaemic control. Undoubtedly, a triple therapy is also associated with increased risk of hypoglycaemia. Therefore, careful monitoring is necessary for safety use of the triple therapy and this is particularly important in T2DM patients because they often have serious concomitant diseases demanding appropriate treatment. Many of drugs used in these patients with comorbidities may significantly affect glucose homeostasis. This situation favours polypragmasy, which is defined as unjustified administration of many drugs when, at least for some of them, there are no evidence for their therapeutic utility. There are, however, reports indicating their potential harmfulness [1]. Unlike combination pharmacotherapy conducted in accordance with medical standards and monitored by the physician, polypragmasy is considered as a serious error. The risk of polypragmasy is increased mainly by lack of knowledge of the drugs' mechanism of action and drug interactions and their potential effects [1].

\section{Advantages of polypharmacy}

The advantage of using polypharmacy over monotherapy has been proven in many common disease entities. For example, in the Angio-Scandinavian Cardiac Outcomes Trial (ASCOT) nearly $90 \%$ of patients with hypertension had to use at least two antihypertensive drugs to reach the target blood pressure values. A metaanalysis of 42 studies including 11,000 hypertensive patients has shown that the additional antihypertensive effect of the combination of drugs from two different groups is almost five-fold higher than doubling the dose of a single drug [2]. 
Fixed-dose combination (FDC) drugs were introduced to improve patients' adherence. These drugs include two or more active agents in fixed doses. The most frequently used FDC drugs include the combinations of two analgesic, hypotensive, antiasthmatic, glucose-lowering and lipid-lowering agents. Successful implementation of FDC drugs into the treatment of civilisation diseases, such as hypertension, atherosclerosis (dyslipidaemias) and diabetes, became an inspiration to the development of a polypill, also called "a superpill". It has been assumed that a tablet containing four substances: an angiotensin converting enzyme inhibitor (ACEI), a beta-blocker, a statin and aspirin would improve patients' adherence and therefore increase the effectiveness of primary prevention of ischaemic disease. It has been estimated that the use of this pill may result in $75 \%$ reduction of cardiovascular events [3]. Data from many clinical trials and meta-analyses indicate that the use of "polypill" improves adherence, decreases blood pressure and favourably modifies lipid profile [4]. Despite these encouraging reports, there are still many doubts concerning effectiveness and safety of FDC drugs in a population of patients aged more than 65 years, including those with T2DM. This refers particularly to drugs with potent pharmacodynamic effect and low therapeutic index.

\section{Limits of polypharmacy}

In elderly patients characterized by comorbidity, polypharmacy is usually unavoidable. With ageing, disorders of biological, cognitive and social functions occur, and the number of reported organ dysfunctions and associated symptoms increases. This results in the increase in the number of administered drugs and, consequently, elevated risk of adverse drug interactions, which not only leads to more frequent hospitalization, but also is a threat to patients' health and life. The cost of the treatment of iatrogenic complications are also increased. Adverse drug-related events are 10-fold more frequent in elderly patients than in younger patients (about $4 \%$ vs. 40\%) [5]. The study of Wang et al. showed that mean number of drugs taken by patients aged $85+$ who were followed-up for 5 years was 9.56 \pm 5.68 , and polypharmacy was observed in as many as $70 \%$ of these patients (the authors adopted cut-off value of $\geq 6$ drugs). At the same time, the authors found that the risk of adverse event increased with the number of drugs taken, regardless of the type of the drug, the age of the patient or concomitant diseases. Furthermore, a 11-year follow-up performed by American researchers from 1995 to 2005 showed that in the study population the risk of iatrogenic complication was increased by $88 \%$ in patients taking 5 or more drugs and by $45 \%$ in patients taking 3 or 4 drugs when compared to those who were administered no more than two drugs [6]. What is the optimal number of drugs, which should not be exceeded? The World Health Organization (WHO) defines polypharmacy as simultaneous use of at least five drugs. This definition is based on the observations that the use of the combination of 2 drugs increases the risk of adverse drug interactions by $5.6 \%$, whereas combination of 5 drugs increases the risk by $50 \%$ and combination of 8 or more drugs - by $100 \%$. This is also consistent with practical experience indicating that the lower number of drugs taken simultaneously, the better.

\section{Reasons of polypragmasy}

Undoubtedly, the most common reason for the use of many drugs in elderly patients is comorbidity and disability. Many believe that the main reason for polypragmasy and its undesirable effect is arbitrary adding by patients over-the-counter drugs (medicinal herbs, diet supplements) to therapies prescribed by the doctor. This significantly increases the risk of complications caused by polypragmasy, considering the fact that most patients do not know the mechanism of action of these drugs and their possible interactions. It is believed that young patients react similarly to the same drug; while in elderly reactions may differ between patients. Liver and renal performance is decreased in elderly patients, which results in altered pharmacokinetics of drugs and increased risk of adverse drug reactions [7].

Unfortunately, physician also contribute to this situation by prescribing their patients another drug without analysing the number and usefulness of the drugs currently taken by the patient. Sometimes physicians introduce symptomatic treatment without extended diagnostics aimed at identification of the underlying cause of the disease or surrender to the pressure of the patient or his/her family.

Due to comorbidity, patients often consult various specialists. This may lead to unwanted duplication of drug, particularly since there are many generics available and a physician may prescribe a formulation containing the same active substance that the patients is already taking [6]. It is important problem, because elderly patients may have trouble understanding complicated medical recommendations or take the same drug several times a day due to memory dysfunction.

Patients over 65 years of age, often burdened with many diseases, are rarely followed-up in clinical trials of new drugs, although they are in many cases the main consumers of new medicinal products. Therefore elderly patients are likely to experience previously unknown serious adverse effects of the therapy [8]. Such situa- 
tions were observed in relations to previously unknown undesirable effects of troglitazone, rosiglitazone, muraglitazar and sibutramine.

The risk of undesirable effects of polypragmasy may also be associated with uncritically following guidelines for treating specific diseases, such as T2DM, usually developed by a narrow group of experts. These guidelines usually refer to the so-called "average patient". Sometimes they do not take into account the individual characteristics of the patient.

\section{Prevention of polypragmasy}

Considering the wide spectrum of causes and the consequences of polypragmasy, all efforts should be made to prevent this phenomenon. At each visit, the physician should check the list of medicines taken by the patient, paying attention to the names of active substances in generic drugs [9]. It is also important to thoroughly collect information about over-thecounter (OTC) medication because patients who are often misled by aggressive advertising campaigns do not consider it advisable to inform his/her doctor about taking OCTs $[10,11]$. When a disease is cured or symptoms are markedly reduced, cessation or modification of currently used therapy should be considered. If possible, one drug should be prescribed instead of several preparations. This applies to drugs that have a beneficial effect on many diseases. A good example is the use of pleiotropic properties of angiotensin converting enzyme inhibitors in patients with T2DM coexisting with coronary artery disease, hypertension and heart failure.

The number of drugs taken by the patient can be reduced by stopping their use in the absence of an expected response, when further treatment is associated with systemic adverse effects. This may be the case for continuing bisphosphonate therapy in patients lying in bed [7].

When discussing the phenomenon of polypragmasy, one should emphasize the existence of the so-called "therapeutic cascade". It concerns the situation when prescribing a particular drug involves the prescription of another drug to avoid adverse effects.

Particularly important in the prevention of the risk of polypragmasy is attributed to the Beers criteria, which describe drug interactions, side effects, and estimates of the cost-benefit ratio for elderly patients. Beers' list of potentially harmful drugs was published in the USA in three successive versions (1991, 1997, 2002). Potentially harmful drugs were define as medicinal products, which use have been found to outweigh the expected clinical benefit and which may be replaced by alternative, better tolerated formulations [12].
A similar list was developed in Germany in 2010 by 38 experts from eight medical specialization areas: geriatrics, clinical pharmacology, family medicine, internal medicine, pain management, neurology, psychiatry and pharmacy. The PRISCUS list (whose name is derived from the Latin term "elder and venerable") provides tips for avoiding drugs that should not be combined, indicates the diseases that require dosage monitoring and laboratory tests that should be done when a drug from the list is prescribed [13]. It should be underlined that classification of the drug as potentially harmful for elderly patients depends equally on availability of alternative agents and the risk of adverse effects caused by a specific drug. Hence, many oral anticoagulants or antiplatelets, which are indispensable in contemporary medicine, will not be qualified a 'potentially harmful' for elderly patients [14].

\section{Under-prescribing}

When analysing polypharmacy and polypragmasy one should not disregard another problem referred as "under-prescribing". Under-prescribing is defined as omission of drugs that the patient should obligatory receive due to his/her clinical condition. In a study performed by Kuijpers et al., the most commonly overlooked drugs were anti-constipation agents in patients chronically taking morphine, beta-blockers in patients after myocardial infarction, and ACEI in patients with heart failure [15]. However, the most difficult problem from the clinical point of view seems to be the use of anticoagulants in elderly patients with atrial fibrillation. Atrial fibrillation affects 9 to $17.6 \%$ of patients over 80 years of age [16]. These patients would meet the criteria for the introduction of anticoagulation therapy based on their age only ( 2 points on $\mathrm{CHA}_{2} \mathrm{DS}_{2}$ VASc for age $\geq$ 75 years). If the risk of bleeding assessed according to the HASBLED scale is not very high, oral anticoagulant therapy is recommended that carries many risks. One should not forget about the phenomenon similar to "under-prescribing", which is "under-dosing" — when a patient is taking a drug at a dose that is too low to be clinically effective. On the other hand, physicians prescribe too low doses of pharmaceuticals because they are afraid of adverse effects of the therapy. Underdosing may be associated with a change in the patient's clinical condition. This can be seen in patients in whom kidney function has improved. In such cases, indications for reducing the dose due to lowered glomerular filtration are now longer applicable. Without adequate dose increase, the expected therapeutic effect may not be obtained. Again, the problem of anticoagulation therapy with coumarin derivatives and the maintenance of INRs within subtherapeutic limits can be raised here 
due to concerns about the risk of overdose of acenocoumarol or warfarin.

\section{Polypharmacy in diabetic patients}

Prospective observational trial performed by Breuker et al. [17] included 904 hospitalized patients of whom 671 had T2DM. It was found at admission that T2DM patients were prescribed twice as many drugs as those without diabetes. During hospitalization, the treatment was assessed, treatment errors detected and the number of drugs for long-term therapy in ambulatory setting reduced. However, at discharge it occurred again that the list of drugs prescribed to T2DM patients was two-fold longer than that of remaining patients! Additionally, T2DM group was also characterized by a higher number of significant therapeutic errors. Based on the results of the analysis, the authors recognized polypharmacy in patients with diabetes (but not diabetes itself!) as an independent risk factor of therapeutic errors. Interestingly, as much as $25.8 \%$ potential drug interaction resulting from inappropriate drug combination were successfully corrected before any harm to the patients occurred. The authors highlight the physicians' responsibility for proper choice of drugs. This is particularly important in elderly patients, including diabetics.

The high proportion of patients with type 2 diabetes also have hypertension requiring treatment. It is known that some of the antihypertensive drugs may increase blood glucose levels and thus interfere with glycaemic control in diabetic patients. It has long been known that beta-blockers and thiazides, especially when used simultaneously, can increase the risk of developing type 2 diabetes. This undesirable phenomenon is caused by increased insulin resistance. On the other hand, it is worth emphasizing that beta-blockers, especially non-cardioselective ones, may increase the severity of hypoglycaemia. Among causes of this phenomenon are the hepatic glucose production and the reduction of glycogenolysis. It is also worth mentioning that beta-blockers can mask the clinical symptoms of hypoglycaemia. On the other hand, ACE inhibitors and sartans have been shown to improve insulin sensitivity [18]. It is suggested that bradykinin plays an important role; its concentration is increased by ACE inhibitors via reducing kinase activity and degrading bradykinin, which in turn leads to vasodilatation and ultimately to increased muscle glucose uptake. Recently, attention has been paid to the risk of disturbing glucose homeostasis by statins. These drugs are very frequently used in patients with type 2 diabetes because of the coexistence of chronic hyperglycaemia with diabetic dyslipidaemia. This has been proven by a meta-analysis by Sattar et al. [19]. Recent data suggest that the risk of developing type 2 diabetes is particularly high in statintreated women over 75 years of age - it is estimated at $33 \%$, and with high doses it increases to $50 \%$ [20]. Many people with diabetes suffer from depression and have to take antidepressants. Some of these drugs, especially the new-generation antidepressants, negatively affect glucose metabolism by increasing insulin resistance [21].

Drugs that promote glucose metabolism disorders are glucocorticoids. The main mechanism responsible for this phenomenon is also the consequence of reducing insulin sensitivity. Clinically important is the interaction of quinolones with glucose metabolism, because it may increase the risk of hypoglycaemia in patients undergoing pharmacotherapy with antidiabetic agents. It is suggested that fluoroquinolones may increase insulin secretion by blocking ATP-sensitive potassium channels [22]. Salicylates affect glucose metabolism via similar mechanism $[23,24]$.

\section{Overtreatment}

Overtreatment consist in unjustified attempts to obtain maximum modification of pathological biochemical changes (e.g. hyperglycaemia) to values close to normal. It has been observed, especially in the elderly with type 2 diabetes who underwent intensive hypoglycaemic therapy that this may result in severe hypoglycaemia. Lipska et al. observed that in many patients in advanced age hypoglycaemic therapy is continued, sometimes with drugs with potent hypoglycaemic effect, although glycated haemoglobin is well below $7 \%$ [25]. This may lead to episodes of severe hypoglycaemia with its dangerous effects. Elderly patients with low levels of $\mathrm{HbA}_{1 \mathrm{c}}$ often do not require any pharmacological treatment at all. Proper diet is sufficient to maintain good glycaemic control. It is worth emphasizing that people in this age category often eat too little, thus providing insufficient amount of carbohydrates, which results in lowering blood glucose.

\section{Conclusions}

Contemporary pharmacology provides broad access to a wide variety of medicines, often available without a prescription. However, it is worth to remember that medicinal products should be used moderately and adequately to current needs of the patient, not duplicated and reliably analysed at every visit. It is very important to maximize benefits and minimize costs of treatment. Each patient should receive individually tailored treatment with the number of drugs adequate to his/her condition. The more drugs, the greater the risk of adverse effects. On the other hand, it is important to remember that under-treatment is also harmful to the patient. 
Elderly patients with diabetes are more likely to have polypharmacy compared to those with normal carbohydrate metabolism. Particular attention should be paid to drugs prescribed to these patients in order to avoid potential therapeutic errors.

How to balance the right proportions? Medicine is art. Since the dawn of history it has been posing the unconventional challenges and searching for solutions. Polypragmasy is one of the important problems of modern medicine, but by eliminating its causes and preventing its effects, we can increase the safety and effectiveness of treatment.

\section{Conflict of interest}

None declared.

\section{The paper was created under the statute UM 503/0-072-09/503-01-006}

\section{REFERENCES}

1. Nobili A, Licata G, Salerno F, et al. SIMI Investigators. Polypharmacy, length of hospital stay, and in-hospital mortality among elderly patients in internal medicine wards. The REPOSI study. Eur J Clin Pharmacol. 2011; 67(5): 507-519, doi: 10.1007/s00228010-0977-0, indexed in Pubmed: 21221958.

2. Wald DS, Law M, Morris JK, et al. Combination therapy versus monotherapy in reducing blood pressure: meta-analysis on 11,000 participants from 42 trials. Am J Med. 2009; 122(3): 290-300, doi: 10.1016/j.amjmed.2008.09.038, indexed in Pubmed: 19272490.

3. Yusuf S. Two decades of progress in preventing vascular disease. Lancet. 2002; 360(9326): 2-3, doi: 10.1016/S01406736(02)09358-3, indexed in Pubmed: 12114031.

4. Webster R, Patel A, Selak V, et al. SPACE Collaboration. Effectiveness of fixed dose combination medication ('polypills') compared with usual care in patients with cardiovascular disease or at high risk: A prospective, individual patient data meta-analysis of 3140 patients in six countries. Int J Cardiol. 2016; 205: 147-156, doi: 10.1016/j.ijcard.2015.12.015, indexed in Pubmed: 26736090.

5. Schäfer I, von Leitner EC, Schön G, et al. Multimorbidity patterns in the elderly: a new approach of disease clustering identifies complex interrelations between chronic conditions. PLoS One. 2010; 5(12): e15941, doi: 10.1371/journal.pone.0015941, indexed in Pubmed: 21209965.

6. Wang R, Chen L, Fan Li, et al. Incidence and Effects of Polypharmacy on Clinical Outcome among Patients Aged 80+: A FiveYear Follow-Up Study. PLoS One. 2015; 10(11): e0142123, doi: 10.1371/journal.pone.0142123, indexed in Pubmed: 26554710.

7. Neumann-Podczaska A, Wawszyk K, Wieczorkowska-Tobis K. Zagrożenia sprawności funkcjonalnej osób starszych wynikające z samoleczenia lekami przeciwbólowymi. Przegląd Lekarski 2012; 69; 10: 773-776.

8. Cruz-Jentoft AJ, Gutiérrez B. Upper age limits in studies submitted to a research ethics committee. Aging Clin Exp Res. 2010; 22(2): 175-178, indexed in Pubmed: 20440104.

9. Ballentine $\mathrm{NH}$. Polypharmacy in the elderly: maximizing benefit, minimizing harm. Crit Care Nurs Q. 2008; 31(1): 40-45, doi:
10.1097/01.CNQ.0000306395.86905.8b, indexed in Pubmed: 18316935.

10. Sobów T. Hazards of polypharmacy in neurology. Postępy Nauk Medycznych. 2010; 23(6): 483-491.

11. Flaherty JH, Takahashi R. The use of complementary and alternative medical therapies among older persons around the world. Clin Geriatr Med. 2004; 20(2): 179-200, v, doi: 10.1016/j. cger.2004.02.003, indexed in Pubmed: 15182877.

12. Lund BC, Steinman MA, Chrischilles EA, et al. Beers criteria as a proxy for inappropriate prescribing of other medications among older adults. Ann Pharmacother. 2011; 45(11): 1363-1370, doi: 10.1345/aph.1Q361, indexed in Pubmed: 21972251.

13. Holt S, SchmiedI S, Thürmann PA. Potentially inappropriate medications in the elderly: the PRISCUS list. Dtsch Arztebl Int. 2010; 107(31-32): 543-551, doi: 10.3238/arztebl.2010.0543, indexed in Pubmed: 20827352.

14. Machalski P, Koziarska-Rościszewska M. Zasady bezpiecznej farmakoterapii osób w starszym wieku — lista PRISCUS. Medycyna Rodzinna. 2013; 3: 106-111.

15. Kuijpers M, Marum Rv, Egberts $A$, et al. Relationship between polypharmacy and underprescribing. British Journal of Clinical Pharmacology. 2008; 65(1): 130-133, doi: 10.1111/j.13652125.2007.02961.x.

16. Fernández C, Formiga F, Camafort M, et al. Erratum: Antithrombotic treatment in elderly patients with atrial fibrillation: a practical approach. BMC Cardiovascular Disorders. 2015; 15(1), doi: 10.1186/s12872-015-0150-x.

17. Breuker C, Abraham O, di Trapanie L, et al. Patients with diabetes are at high risk of serious medication errors at hospital: Interest of clinical pharmacist intervention to improve healthcare. Europ J Inter Med. 2017; 38: 38-45.

18. Singh B, Mourya A, Sah SP, et al. Protective effect of losartan and ramipril against stress induced insulin resistance and related complications: Anti-inflammatory mechanisms. Eur J Pharmacol. 2017; 801: 54-61, doi: 10.1016/j.ejphar.2017.02.050, indexed in Pubmed: 28259714.

19. Sattar NA, Ginsberg H, Ray K, et al. The use of statins in people at risk of developing diabetes mellitus: evidence and guidance for clinical practice. Atheroscler Suppl. 2014; 15(1): 1-15, doi: 10.1016/j.atherosclerosissup.2014.04.001, indexed in Pubmed: 24840509.

20. Jones M, Tett S, Peeters GM, et al. New-Onset Diabetes After Statin Exposure in Elderly Women: The Australian Longitudinal Study on Women's Health. Drugs Aging. 2017; 34(3): 203-209, doi: 10.1007/s40266-017-0435-0, indexed in Pubmed: 28138911.

21. Semenkovich K, Brown ME, Svrakic DM, et al. Depression in type 2 diabetes mellitus: prevalence, impact, and treatment. Drugs. 2015; 75(6): 577-587, doi: 10.1007/s40265-015-0347-4, indexed in Pubmed: 25851098.

22. Teifer SJ. Fluoroquinolone antibiotics and type 2 diabetes mellitus Med Hypotheses. 2014 Sep.; 83(3): 263-9, doi: 10.1016/j. mehy.2014.05.013, indexed in Pubmed: 24947193.

23. Rena G, Sakamoto K. Salicylic acid: old and new implications for the treatment of type 2 diabetes? Diabetol Int. 2014; 5(4): 212-218, doi: 10.1007/s13340-014-0177-8, indexed in Pubmed: 27656338.

24. Mays HV, Setter SM. P Drug-induced glucose alternations part 1: Drug-induced hypoglycemia Diabetes spectrum 2011; Vol. 24, no. 3: 171.

25. Lipska KJ, Krumholz H, Soones T, et al. Polypharmacy in the Aging Patient: A Review of Glycemic Control in Older Adults With Type 2 Diabetes. JAMA. 2016; 315(10): 1034-1045, doi: 10.1001/ /jama.2016.0299, indexed in Pubmed: 26954412. 